\title{
Phase-Change Band-Pass Filters for Multispectral Imaging
}

\author{
Liam Trimby*, Anna Baldycheva, C David Wright \\ Department of Engineering, University of Exeter, EX4 4QF, United Kingdom
}

\begin{abstract}
Phase-change materials (PCMs) provide a route to adding dynamic tunability and reconfigurability to many types of photonic devices by changing the phase-state of the PCM itself. In this work we discuss the use of the phase-change alloy GeSbTe (GST) in the design of dynamically tunable filters operating in the infrared. GST is used to manipulate the extraordinary optical transmission of a periodic hole-array in a metallic layer, so creating ultra-thin, tunable band-pass filters. We discuss the use of such filters for multispectral imaging, suggest some approaches to overcome various practical challenges, and, finally, show that through the use of appropriate post processing algorithms this tunable filter could provide a cheap, ultra-thin, real-time, and relatively high performance multispectral imaging device.
\end{abstract}

Keywords: Metamaterial, Phase-change material, Tunable filter, Multispectral Imaging

\section{INTRODUCTION}

A periodic array of sub-wavelength holes (metamaterial) in a thin metal film will show high transmission for a narrow wavelength band, with other wavelengths being reflected. This phenomenon is named extraordinary optical transmission (EOT). In this work, such a hole-array is coated in a phase-change material (PCM), whose refractive index changes the wavelength at which incident light can couple into the metallic hole-array. Since the refractive index of the PCM can be altered by changing its phase-state (i.e. switching it between amorphous and crystalline, or partially crystalline, states), the inclusion of a PCM layer enables the EOT transmission peak to be dynamically tuned (shifted) in wavelength.

The use of phase-change materials in conjunction with metamaterials to add even greater control over light has experienced growing interest, and shows great promise for a wide variety of applications such as beam-steering, displays, absorbers, filters and more ${ }^{1,2}$. These materials are stable in both amorphous and crystalline states, with such states possessing distinctly different opto-electrical properties. We can rapidly switch between these states by applying heat. Critical to this work is the large change in refractive index obtained in GeSbTe alloys (here $\mathrm{Ge}_{3} \mathrm{Sb}_{2} \mathrm{Te}_{6}$ ), as well as the fact that intermediate crystalline/amorphous states exist, with correspondingly intermediate properties ${ }^{3,4}$. Other approaches to tuning EOT have been explored, such as applying magnetic fields ${ }^{5}$, which may also be compatible with PCMs.

Unlike a typical image which contains intensity data for three wavelength bands (RGB), a multispectral image contains data for up to around a dozen wavelength bands. Multispectral images provide greater contrast between different materials and have wide ranging applications including medical diagnosis ${ }^{6}$, sorting recycling ${ }^{7}$, defeating camouflage, and data-dense satellite mapping ${ }^{8}$. These images are typically taken using a disc of fixed wavelength filters which rotate in time with a camera's capture rate, or by combining multiple sensors. These methods are both bulky and relatively expensive. Another approach to multispectral imaging involves tunable band-pass filters, which have historically been based on technologies like liquid crystals or rotating prisms ${ }^{9}$; however, these have wavelength restrictions and can be quite slow.

A tunable band-pass filter can be used to take a multispectral image by placing it in the optical path of a broadband camera and taking a picture for every state of the tunable filter. The speed at which the filter can be re-tuned determines how long (how many camera frames) it takes to build up a multispectral image (in most cases it is advantageous for the filter bandwidths to partially overlap, as this can be used to improve the spectral resolution via post-processing). The ability of PCM-based filters to be rapidly and continuously tuned over their entire spectral range makes them particularly attractive for fast, high-resolution multispectral imaging. 


\subsection{Background - Extraordinary optical transmission}

Extraordinary optical transmission (EOT) is a phenomenon in which transmission through a metallic film perforated with holes is significantly enhanced around a particular resonant wavelength. EOT was first reported in $1998^{10}$. It has taken many years to fully understand the phenomenon due to the large number of effects which contribute towards the high transmission, with such contributions depending on the wavelength regime. There are also many forms of EOT which depend on the nature of the hole distributions (isolated, random, periodic) and whether the metal layer is smooth or has been selectively corrugated to boost performance ${ }^{11}$. Here we will focus on a smooth, optically thick film, with periodically distributed holes, often referred to as surface-enhanced EOT.

The resonant wavelength is primarily determined by the index of the substrate, and the hole spacing. For the case where there is only air above the metal, the transmission peak occurs at a slightly longer wavelength than the Rayleigh wavelength $\lambda_{R}=n_{s} P$ where $n_{s}$ is the substrate index and $P$ is the hole spacing. The primary mechanism for this transmission involves surface plasmon polaritons present at the dielectric-metal interfaces. Typically these plasmons cannot be excited by incident radiation as both momentum and energy would not be conserved. The holes modify the surface mode relaxing the momentum conservation, and allowing incident light to couple into the plasmons, meaning light that would have been reflected remains at the dielectric-metal interface and builds up a number of transmission resonances, with a portion radiating out the backside of the holes ${ }^{12,13}$.

The transmission is greatest when the surface modes at both the substrate and superstrate occur for the same wavelength ${ }^{14}$ (i.e. matched indices either side of metal), and if the holes are filled with a high index dielectric. In the case of our PCM tunable filter, the indices could be matched by sandwiching the metal layer between two PCM layers. However, because the PCM is lossy it was found to be more effective to remove the lower PCM layer and slightly thicken the top layer.

\subsection{Background - Phase-change materials}

Phase-change materials can stably exist in either a glassy amorphous phase, or an ordered crystalline phase, and possess the ability (via thermal, electrical or optical excitations) to quickly and reversibly switch between phases. These phases exhibit high contrast in many properties, with optical and electrical properties being of most interest for providing tunability to optoelectronic devices. Typically the crystalline phase will exhibit substantially greater thermal/electrical conductivity and (in the infrared) higher refractive index (n) and optical losses (k) as compared to its amorphous counterpart

There are numerous phase-change alloys, a few of which have seen success in commercial products. The most widely used and well researched is an alloy of germanium, antimony, and tellurium, $\mathrm{Ge}_{2} \mathrm{Sb}_{2} \mathrm{Te}_{5}$, as used in many re-writable optical disc formats. This work uses a variation of this alloy, $\mathrm{Ge}_{3} \mathrm{Sb}_{2} \mathrm{Te}_{6}$, henceforth referred to as GST-326, which has particularly attractive (for our target application) infrared optical properties. As shown in figure 1, over the wavelength range of 2 to $12 \mu \mathrm{m}$, the refractive index approximately doubles when crystallized, providing excellent tunability. Amorphous GST-326 is effectively transparent from about $1.5 \mu \mathrm{m}$ onwards, and although the crystalline phase is substantially more absorbing, in the mid and long wave infrared it remains useable in transmission devices provided thicknesses are kept low.

The switching of the PCM between states is achieved by controlling the material's temperature, usually through electrical or optical heating. Importantly, the phase-states are non-volatile and, after switching, the PCM remains in its switched state for a very long time (years at room temperature). To switch to the crystalline phase the material must be held between its crystallization and melting temperatures for a brief period of time corresponding to the temperature applied, see figure 2. This can be on the order of nanoseconds ${ }^{4}$, but for multispectral imaging slower tuning speeds (and so lower excitation temperatures) are more than adequate (since speeds will be determined by camera frame rates, which are relatively slow). The crystallization process can be halted at any time by bringing the temperature back into the stable region (see figure 2), and this results in a partially crystalline material with correspondingly intermediate (between fully crystalline and fully amorphous) properties. To transition back from crystalline to amorphous the material must be brought above its melting temperature then rapidly quenched; this melt quench transition is much faster than crystallization, although achieving the required cooling rates can be practically challenging within devices.

Phase-change materials have excellent switching cycle endurance provided they are suitably encapsulated to protect from oxidation $^{15}$. Aside from capping layers to protect from the atmosphere, barrier layers are also required when in contact with some metals such as gold, as GeSbTe alloys readily mix with many metals at elevated temperatures. Finally, the volume of GeSbTe phase-change alloys reduces by approximately 8 to $15 \%$ (dependent on composition) when fully 
crystalline, and any barrier and encapsulation layers must be able to cope with the stresses induced due to such volume changes.
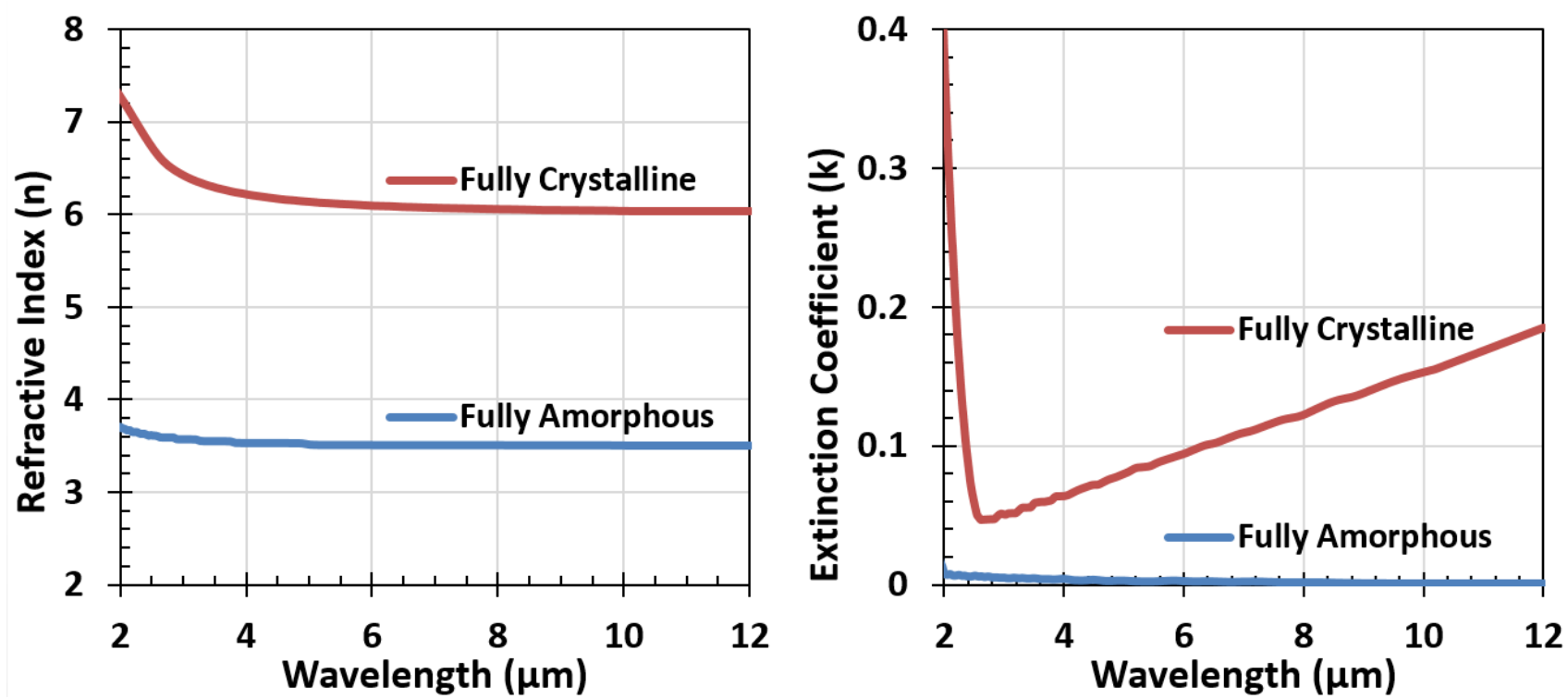

Figure 1. Optical properties of $\mathrm{Ge}_{3} \mathrm{Sb}_{2} \mathrm{Te}_{6}$ in both amorphous and crystalline phases ${ }^{16}$.
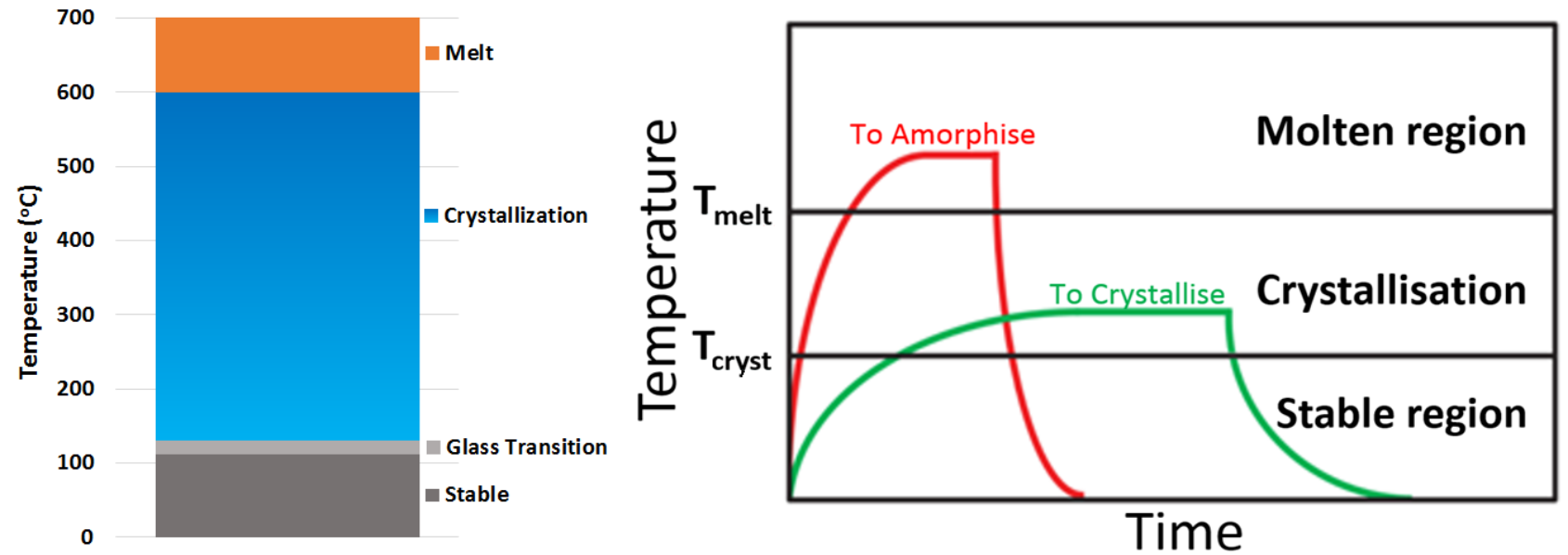

Figure 2. Left: Thermal properties of GST-326. Crystallization occurs between approximately $130^{\circ} \mathrm{C}$ and $600^{\circ} \mathrm{C}$ (depending on deposition method and composition $)^{17}$ Right: Typical temperature profiles for switching: to amorphize the PCM must be molten, and then rapidly quenched; to crystallize the PCM must be held at a temperature within its crystallization region.

An important consideration when designing devices which incorporate a PCM with in-situ switching is that films must be kept thin, typically sub-100 $\mathrm{nm}$. This is so the entire volume can be brought to the required temperature without a significant temperature gradient caused by the (relatively) low thermal conductivity of the PCM. This especially applies when quenching from the molten state, as this must occur very rapidly. 


\section{PCM BAND-PASS FILTER STRUCTURE AND PERFORMANCE}

\subsection{Filter structure}

The filter, as shown in figure 3, consists of an array of subwavelength holes within a metal layer, which is then coated in a phase-change material, and finally capped in a thin layer of protective transparent material to protect the PCM layer from oxidation. The capping layer also serves a role in mitigating the effects of the volume change between amorphous and crystalline states, and prevents the PCM from flowing into the holes during the brief molten stage required for reamorphization. The substrate can be any transparent material, and this kind of filter could potentially be fabricated directly onto a lens.

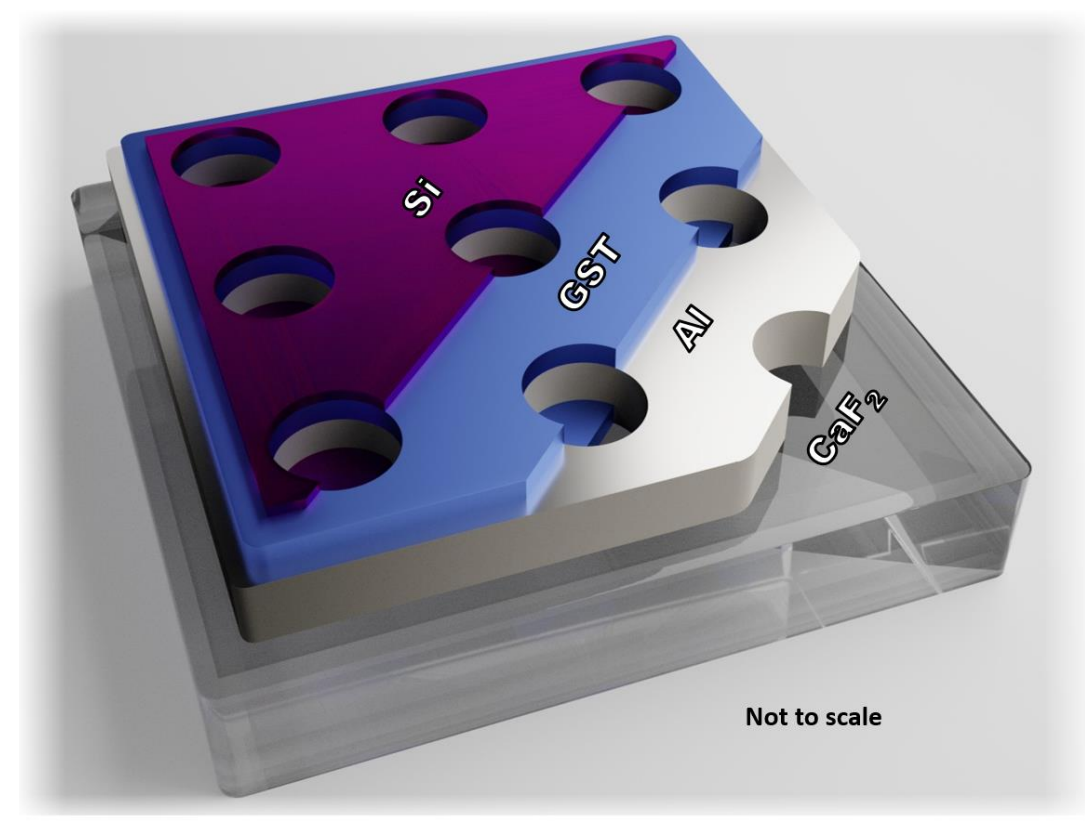

Figure 3. Rendered illustrative image of PCM band-pass filter device. Not to scale.

\subsection{Filter performance}

The performance of this filter was simulated using COMSOL 5.3 for two designs, one for each major atmospheric transmission window. The first filter, designed for the MWIR, was able to cover the vast majority of the 3 to $5 \mu \mathrm{m}$ atmospheric window with a GST-326 layer of $70 \mathrm{~nm}$ thickness. The second filter, designed for the LWIR, covers approximately two thirds of the 8 to $12 \mu \mathrm{m}$ long wave atmospheric window using a $120 \mathrm{~nm}$ GST-326 layer.

Figure 4 shows the simulated transmission spectra for both the MWIR and LWIR filters, for the PCM layer in both the fully amorphous and fully crystalline states (i.e. $0 \%$ and $100 \%$ crystalline). It can be seen that the Q-factor and peak height are reduced for greater crystalline fractions in the filter. This is because the losses of the PCM significantly increase when crystalline. The background noise far from resonance is just a few percent, which yields an SNR of around 30 for a filter in the amorphous state and 18 for $100 \%$ crystalline.

As summarized in Table 1, there are a number of ways to modify the filter performance, depending on requirements, although improving one aspect of the filter performance often adversely affects another. Typically, increasing Q-factor or spectral range will come at the cost of peak transmission, and vice-versa. The substrate refractive index also plays a significant role. With a lower index substrate, losses due to reflection at the air interface decrease, and also blue shifting 
of the peak occurs. Although generally for EOT it is desirable to match the substrate and superstrate index, in this case the GST-326 superstrate is extremely thin, and so a low index substrate yields superior performance.
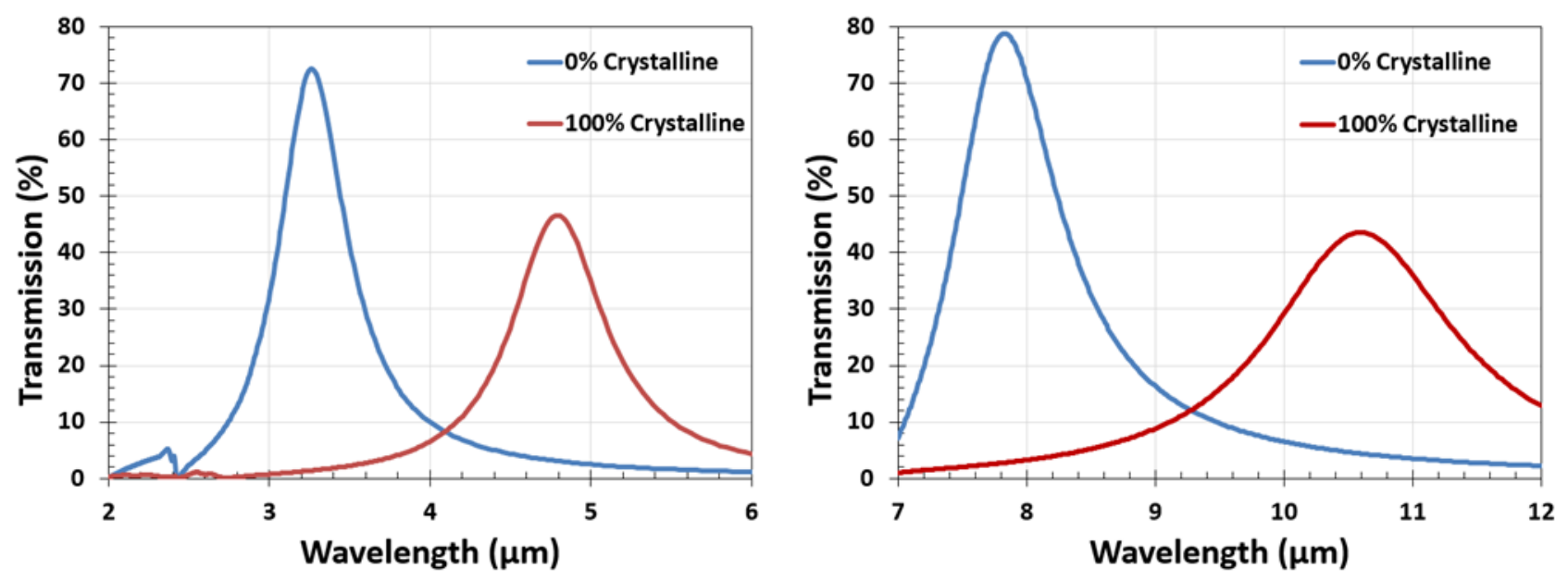

Figure 4. Simulated transmission of tunable filter for the fully amorphous and fully crystalline states. Intermediate crystalline states are not shown, but would be present during actual application. Left: Filter designed for 3-5 $\mu \mathrm{m}$ range. Right: For 8-12 $\mu \mathrm{m}$ range.

Table 1. Summary of filter geometrical parameters and their affect upon performance

\begin{tabular}{|l|l|l|l|l|}
\hline Geometry & Transmission \% & Q-Factor & Spectral Range & Peak Position \\
\hline Thicker PCM layer & Reduced & Reduced & Increased & Red-shift \\
\hline Thicker metal layer & Reduced & Increased & Unchanged & Blue-shift \\
\hline Hole Diameter & Increased & Reduced & Unchanged & Red-shift \\
\hline Larger Hole spacing & Unchanged & Unchanged & Unchanged & Red-shift \\
\hline
\end{tabular}

\subsection{Tuning the filter}

In-situ crystallization of GeSbTe-based PCM layers in similar devices to that shown in figure 4 has been previously demonstrated using the metal layer as a heater and passing a current through it ${ }^{18}$. However, it is not clear that the cooling rates required for re-amorphization could be practically achieved with this approach, due to the relatively thick PCM layer needed to spectrally cover an entire atmospheric transmission window. If only a narrow spectral range is needed, then a thinner PCM layer could be used (10 to $20 \mathrm{~nm}$ ), which should be re-amorphizable using the metal layer as a heater. An alternative switching approach could be to use the high absorption of GST-326 in the visible region, and focus a laser/LED onto the filter surface to provide the required heating.

\section{MULTISPECTRAL IMAGING USING THE PCM BAND-PASS FILTER.}

\subsection{Imaging method}

To produce a multispectral image the filter would be placed in the optical path between the scene to be imaged and a broadband camera sensitive in the operating range of the filter, as shown in figure 5A. As the PCM layer within the filter is heated and crystallizes, the transmission peak will shift from shorter to longer wavelengths (as was shown in figure 4). While this is occurring, the camera takes a number of images (figure 5B), with each image corresponding to a particular 
time-step since crystallization began, and therefore a particular crystallization state and filter response (figure 5C). Figure 5 shows this process for the case where the camera takes six images during the crystallization process.

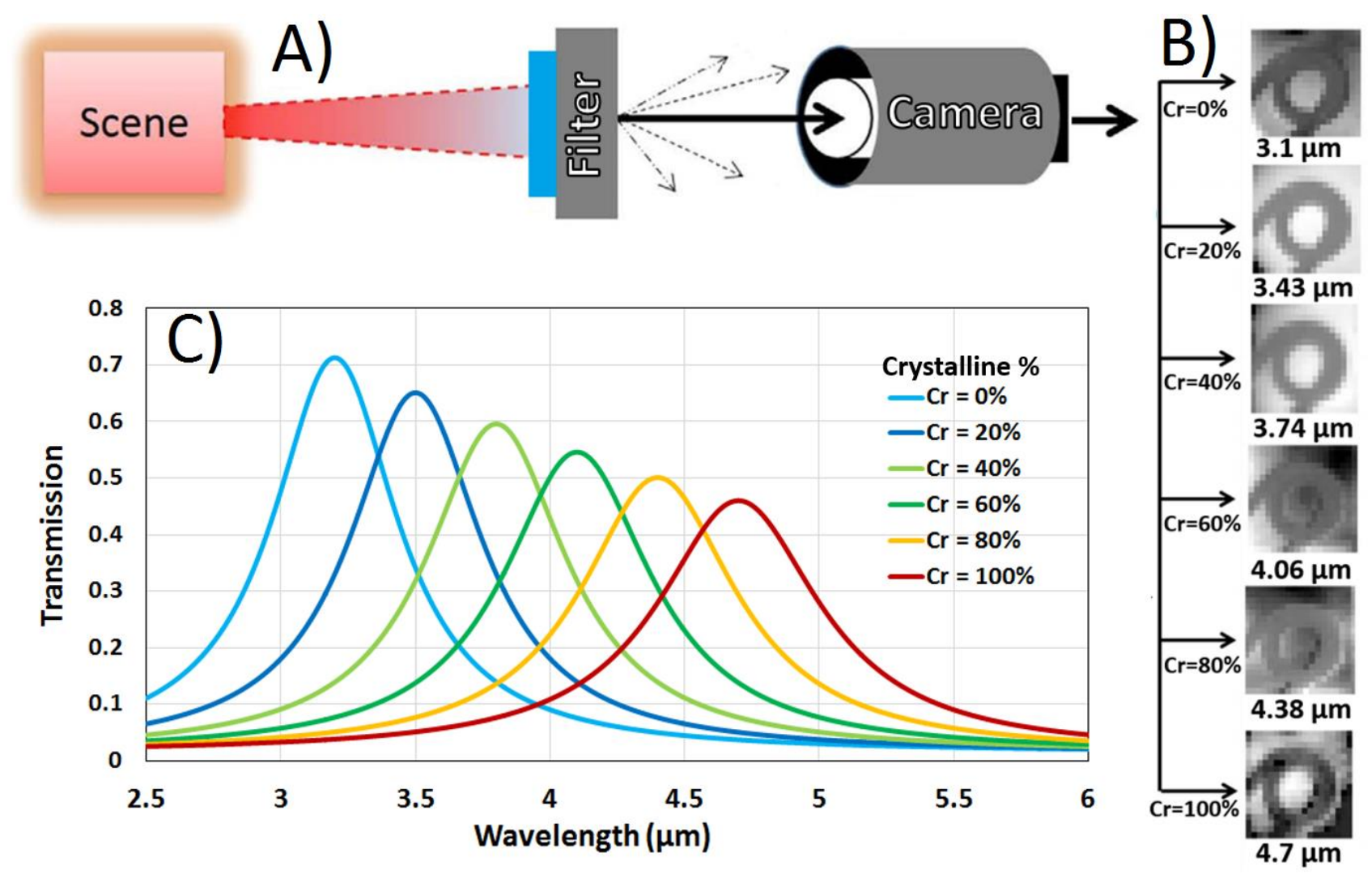

Figure 5. An overview of the imaging method given that the camera takes six pictures during the crystallization process. A) Illustrative image of imaging setup, with the filter in the optical path of a broadband camera. B) Images taken of a hot iron ring through a number of similar but non-tunable filters (taken from ref. [19] and used here for illustrative purposes only). C) PCM band-pass filter responses at evenly spaced crystalline percentages.

Building on the example in figure 5, the raw data from this image would be six intensity values per pixel, and each of these intensity values would have a corresponding filter response curve. Assuming that the filter response is known for all six values, then the overlapping nature of the filter's response can be exploited to resolve much smaller wavelength intervals than might be expected given the Q-factor of each individual peak. This could be achieved, for example, by using a weighted linear combination of all of the overlapping responses ${ }^{19}$. Infrared detectors are typically starved for photons, and so the transmission for the higher crystalline fractions may be lower than desired. The transmission can be increased, for the same spectral range, by enlarging the holes or by thinning the metal, which broadens the peaks and increases background noise respectively. However, this can be somewhat compensated for by using a higher frame-rate camera. This is because the resolution of the reproduced spectra after post processing is dependent upon not only the Q-factor of the filter's transmission peaks, but also how many total data points there are available.

\subsection{Tuning considerations}

A challenge to practically realizing multispectral imaging with a PCM band-pass filter will be that of characterizing the filter response as a function of time (rather than crystallization percentage). This characterization will be essential to correctly match each camera frame to the correct transmission response. Careful calibration of the time-temperature crystallization properties, and accurate control and in-situ measurement of the temperature experienced by the PCM-layer, are likely to be needed to overcome this challenge. 
Another challenge will be mitigating thermal noise from the filter itself during the crystallization process (as this requires heat). This means care must be taken when selecting an appropriate crystallization temperature, so as not to produce high noise levels in the desired detection range (see figure 6). Ideally the crystallization rate should be relatively low (millisecond range), so allowing for relatively low excitation temperatures to be used. In practice it will be important to choose a crystallization temperature which emits at a peak wavelength far from the filter's operational range. For the MWIR design a low temperature around $130^{\circ} \mathrm{C}$ can be used, while for the LWIR design a higher temperature of at least $200^{\circ} \mathrm{C}$ will be preferable.

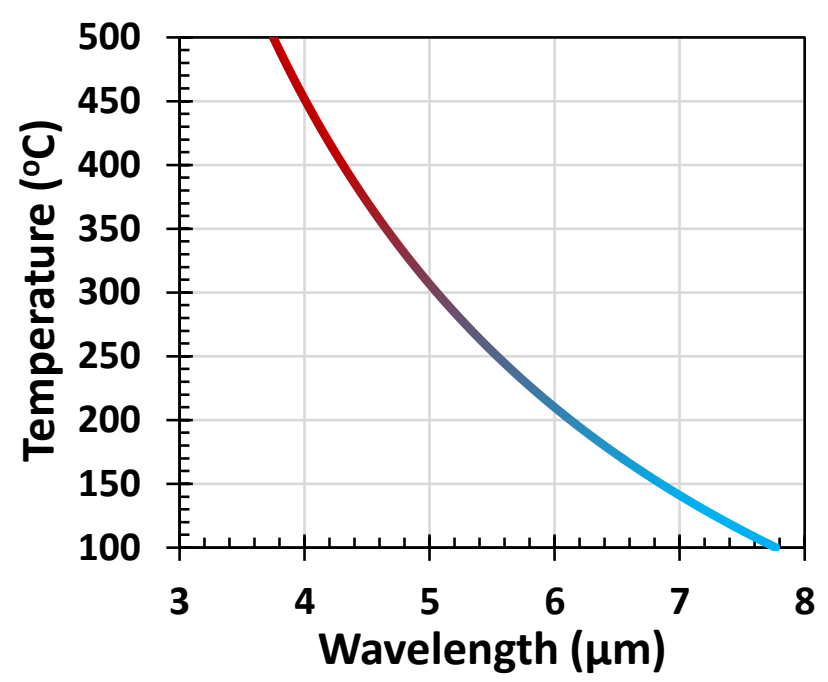

Figure 6. Peak black body emission wavelength vs. temperature. Calculated with Wien's Displacement Law.

\section{CONCLUSION}

We have proposed a novel approach to creating dynamically tunable band-pass filters by combining periodic metallic holearrays and phase-change materials. These filters show competitive performance in simulations, will work for many wavelength regions in which a suitably low-loss PCM is available, are ultra-thin, and have easily modifiable performance through the selection of appropriate device geometries. The wide wavelength range of these PCM-based filters could allow them to fill important niches, such as the LWIR range, in which existing technologies (such as liquid crystal based filters) cannot operate. The compact design and low film thicknesses also make the PCM-based filters well-suited to weightcritical applications. There is also scope to further improve their optical performance by moving to designs with more complex corrugated film structures. Moreover, PCM-based band-pass filters can be dynamically tuned within nanoseconds if required, and due to the continuous tuning nature should be well suited to ultra-fast multispectral imaging applications when used with a high-speed camera and post-processing algorithms. We have also addressed a number of drawbacks to this approach, most of which arise mostly from the properties of the PCM itself. It now remains to fabricate these filters and ensure that the challenges presented can be overcome in practice.

\section{ACKNOWLEDGEMENTS}

CDW acknowledges funding via the US Naval Research Laboratories ONRG programme (\#N62909-16-1-2174) and the EPSRC ChAMP and WAFT grants (EP/M015130/1 and EP/M015173/1). LT acknowledges funding via the EPSRC CDT in Metamaterials(EP/L015331/1) and via QinetiQ PLC

\section{REFERENCES}

1. Wuttig, M., Bhaskaran, H. \& Taubner, T. Phase-change materials for non-volatile photonic applications. Nat Photonics 11, 465-476 (2017).

2. Raeis-Hosseini, N. \& Rho, J. Metasurfaces based on phase-change material as a reconfigurable platform for multifunctional devices. Materials (Basel). 10, (2017).

3. Wright, C. D., Hosseini, P. \& Diosdado, J. a V. Beyond von-neumann computing with nanoscale phase-change memory devices. Adv. Funct. Mater. 23, 2248-2254 (2013). 
4. Bruns, G. et al. Nanosecond switching in GeTe phase change memory cells. Appl. Phys. Lett. 95, (2009).

5. Battula, A., Chen, S., Lu, Y., Knize, R. J. \& Reinhardt, K. Tuning the extraordinary optical transmission through subwavelength hole array by applying a magnetic field. Opt. Lett. 32, 2692-2694 (2007).

6. Liu, W.-L. et al. Application of multispectral imaging in quantitative immunohistochemistry study of breast cancer: a comparative study. Tumor Biol. 5013-5024 (2015). doi:10.1007/s13277-015-4327-9

7. Bank, W. \& Korea, S. How Multispectral and Hyperspectral Imaging are Improving Recycling. (2013).

8. Coffey, V. C. Multispectral Imaging Moves into the Mainstream. Opt. Photonics News 23, 18 (2012).

9. Gat, N. Imaging spectroscopy using tunable filters: a review. Proc. SPIE 4056, 50-64 (2000).

10. Ebbesen, T. W. et al. Extraordinary optical transmission through sub-wavelength hole arrays. Nature 86, 1114-7 (1998).

11. Ishi, T., Fujikata, J. \& Ohashi, K. Large optical transmission through a single subwavelength hole associated with a sharp-apex grating. Japanese J. Appl. Physics, Part 2 Lett. 44, (2005).

12. Rodrigo, B. S. G. \& Leo, F. De. Transmission : Fundamentals and Applications. 104, (2016).

13. Rodrigo, S. G. Optical Properties of Nanostructured Metallic Systems. (2012). doi:10.1007/978-3-642-23085-1

14. Krishnan, A. et al. Evanescently coupled resonance in surface plasmon enhanced transmission. Opt. Commun. 200, 1-7 (2001).

15. Berthier, R. et al. In situ observation of the impact of surface oxidation on the crystallization mechanism of GeTe phase-change thin films by scanning transmission electron microscopy. J. Appl. Phys. 122, (2017).

16. Michel, A. K. U., Wuttig, M. \& Taubner, T. Design Parameters for Phase-Change Materials for Nanostructure Resonance Tuning. Adv. Opt. Mater. 5, 1-8 (2017).

17. Kalb, J. a., Wuttig, M. \& Spaepen, F. Calorimetric measurements of structural relaxation and glass transition temperatures in sputtered films of amorphous Te alloys used for phase change recording. J. Mater. Res. 22, 748 754 (2007).

18. Rudé, M. et al. Ultrafast and Broadband Tuning of Resonant Optical Nanostructures Using Phase-Change Materials. Adv. Opt. Mater. 4, 1060-1066 (2016).

19. Jang, W.-Y. et al. Experimental Demonstration of Adaptive Infrared Multispectral Imaging using Plasmonic Filter Array. Sci. Rep. 6, 34876 (2016). 